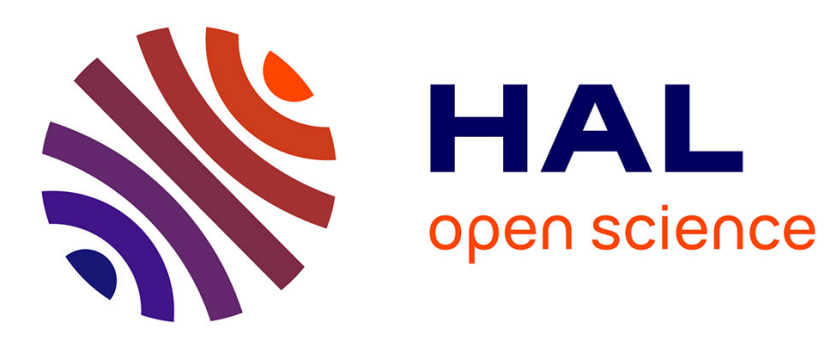

\title{
Numerical construction of "optimal" nonoscillating amplitude and phase functions
}

\author{
A. Matzkin, M. Lombardi
}

\section{To cite this version:}

A. Matzkin, M. Lombardi. Numerical construction of "optimal" nonoscillating amplitude and phase functions. Physical Review E: Statistical, Nonlinear, and Soft Matter Physics, 2002, 66, pp.37702. 10.1103/PHYSREVE.66.037702 . hal-00961319

\section{HAL Id: hal-00961319 \\ https://hal.science/hal-00961319}

Submitted on 19 Mar 2014

HAL is a multi-disciplinary open access archive for the deposit and dissemination of scientific research documents, whether they are published or not. The documents may come from teaching and research institutions in France or abroad, or from public or private research centers.
L'archive ouverte pluridisciplinaire HAL, est destinée au dépôt et à la diffusion de documents scientifiques de niveau recherche, publiés ou non, émanant des établissements d'enseignement et de recherche français ou étrangers, des laboratoires publics ou privés. 


\title{
Numerical construction of "optimal" nonoscillating amplitude and phase functions
}

\author{
A. Matzkin ${ }^{1,2}$ and M. Lombardi ${ }^{2}$ \\ ${ }^{1}$ Department of Physics and Astronomy, University College London, Gower Street, London WClE 6BT, United Kingdom \\ ${ }^{2}$ Laboratoire de Spectrométrie Physique, CNRS and Université Joseph-Fourier Grenoble-I, Boîte Postale 87, \\ F-38402 Saint-Martin, France
}

(Received 17 January 2002; revised manuscript received 23 May 2002; published 30 September 2002)

\begin{abstract}
A numerical recipe for the construction of nonoscillating amplitude and phase functions for potentials with a single minimum is given. We give different examples illustrating the recipe, showing the usefulness of the procedure for the construction of basis functions in bound-state scattering processes, such as those described by quantum defect theory. The resulting amplitude and accumulated phase functions are coined as "optimal" nonoscillating (as a function of the space and energy variables) because they are the counterpart for the quantum problem of the classical action for the analog semiclassical problem.
\end{abstract}

DOI: 10.1103/PhysRevE.66.037702

PACS number(s): 02.60.Lj, 03.65.Ge, 31.15.-p

\section{INTRODUCTION}

The interest in amplitude-phase methods for solving bound-state scattering problems is well known [1-4]. The advantage of using the amplitude-phase formulation is that the oscillatory character of the wave functions is obtained from amplitude and phase functions [respectively, denoted $\alpha(x, E)$ and $\phi(x, E)]$ which are better behaved (ideally, nonoscillating) in terms of the space and energy variables. Let $\psi(x, E)$ be a solution of the Schrödinger equation

$$
\partial_{x}^{2} \psi(x)+k^{2}(x) \psi(x)=0,
$$

with $k^{2}(x)=2[E-V(x)]$, where $V(x)$ is a potential well with a single minimum defined on an interval $] s_{1}, s_{2}[$. The same wave function may be written as

$$
\psi(x)=\alpha(x) \sin [\phi(x)]
$$

in terms of any set of functions $\alpha$ and $\phi$ solutions of the so-called Milne equation [5]

$$
\partial_{x}^{2} \alpha(x)+k^{2}(x) \alpha(x)=\alpha^{-3}(x),
$$

with $\alpha^{-2}(x)=\partial_{x} \phi(x)$. Although the direct integration of Eq. (3) has been used as an efficient manner of solving the Schrödinger equation, amplitude-phase methods have mainly been employed when a particle is subjected to distinct shortrange and long-range interactions, as is the case for an atomic or molecular Rydberg electron. Then $\alpha$ and $\phi$ are often obtained from known solutions of Eq. (1) in the longrange potential; the total wave function including the shortrange potential is at last determined from $\alpha, \phi$, and the relevant energy-dependent phase shifts, provided these functions are smooth. The problem is that for arbitrary boundary conditions, $\alpha$ is highly oscillatory, and the quantity $\beta$ $\equiv \phi\left(s_{2}\right)-\phi\left(s_{1}\right)$ known as the accumulated phase and which defines the normalization of $\psi$ oscillates as a function of $E$. This is why there has been renewed interest in devising numerical methods aiming at minimizing these oscillations [6-10]. This is also important in other problems employing the amplitude-phase formalism, such as the parametric timedependent oscillator in classical mechanics [11], and is of potential interest to other areas of physics where the Milne equation appears (e.g., in the analysis of supersymmetric families of damping modes [12], or in studies of the gravitational equilibrium of stellar structures [13]).

The present work introduces a numerical method very simple to implement, which leads to the construction of optimal amplitude and phase functions. By "optimal" we mean here that the amplitude function is nonoscillating in the space variable and that the accumulated phase is a monotonic function of the energy. These nonoscillating properties stem from a remarkable feature, recently shown by one of us [14]: there is only a single function $\phi_{o}(x, E)$ which in the limit $\hbar \rightarrow 0$ tends to the reduced classical action $S(x, E)$ [and concurrently $\left.\alpha_{o}(x, E) \rightarrow k^{-1 / 2}(x, E)\right] . \alpha_{o}$ is then a nonoscillating function of $x$, and $\beta_{o}$ is a monotonic function of $E$. For other choices of $\phi$ or $\alpha$, highly oscillatory semiclassical phase and amplitude functions $\widetilde{\phi}$ and $\widetilde{\alpha}$ are obtained when the limit $\hbar$ $\rightarrow 0$ is taken. We give below a recipe for the practical construction of $\alpha_{o}$ and $\phi_{o}$, after recalling the mathematical framework. We then work out this recipe in three different cases, for which the implementation of the method slightly differs.

\section{BACKGROUND}

We briefly review for reference the amplitude-phase formulation within the context of Ermakov systems [Eqs. (1) and (3) form together a system known as an uncoupled Ermakov system; for details we refer the reader to Refs. [8,14] and references therein]. Labeling $u_{1}$ and $u_{2}$ two independent solutions of Eq. (1) with Wronskian $W \equiv\left(\partial_{x} u_{1}\right) u_{2}$ $-u_{1}\left(\partial_{x} u_{2}\right)$, the general solution of Eq. (3) takes the form

$$
\alpha(x)=\left[\left(\frac{1}{2 I}+2 I c^{2}\right) u_{1}^{2}(x)+\frac{2 I}{W^{2}} u_{2}^{2}(x)-\frac{4 I c}{W} u_{1}(x) u_{2}(x)\right]^{1 / 2}
$$

and the equation for $\phi$ is integrated as

$$
\phi(x)=\arctan \left[\left(\frac{1}{2 I}+2 I c^{2}\right) W \frac{u_{1}(x)}{u_{2}(x)}-2 I c\right]+\arctan 2 I c,
$$


with $\phi\left(s_{1}\right)=0$. I and $c$ are two integration constants that set the boundary conditions of Eq. (3). For quantized energies $E=E_{0}$, the accumulated phase is

$$
\beta\left(E=E_{0}\right)=n \pi,
$$

where $n$ in an integer counting the number of halfwavelengths between $s_{1}$ and $s_{2}$. The eigenfunctions are then properly normalized,

$$
\int_{s_{1}}^{s_{2}} \psi^{2}\left(x, E_{0}\right) d x=I \partial_{E} \beta\left(E_{0}\right)=I \pi \partial_{E} n
$$

where $n\left(E_{0}\right)$ gives the functional relation between the number of half-wavelengths and the energy. For $E \neq E_{0}, \beta$ depends on the boundary conditions,

$$
\beta\left(E \neq E_{0}\right)=\arctan (2 I c)+\frac{(2 n+1)}{2} \pi,
$$

and a solution of Eq. (1) is improperly normalized as

$$
\int_{r}^{s_{2}} \psi^{2}(x, E) d x=I \partial_{E} \beta(E)=I\left[\partial_{E}(2 I c)\right]\left(1+4 I^{2} c^{2}\right)^{-1}
$$

$r$ is a cutoff radius, since $\psi$ diverges at $s_{1}$, and thus improper normalization is arbitrary, depending on $I$ and $c$. It follows from Eq. (7) that $I$ can take two meaningful values: $I$ $=\pi^{-1}$ if the eigenfunctions are energy normalized, or $I$ $=\left(\pi \partial_{E} n\right)^{-1}$ if the functions are unity normalized. We shall assume energy normalization since it is the case most commonly encountered in practice. The constant $c$ is set by requiring improper normalization to be defined, for any $E$, by the same functional dependence as for $E=E_{0}$. This gives $c$ $=-\pi \cot \pi n(E) / 2$ and $\beta(E)=\pi n(E)$, where $n(E)$ is a real (noninteger) number and the right hand side of Eq. (9) simply becomes $I \pi \partial_{E} n$.

\section{NUMERICAL CONSTRUCTION OF NONOSCILLATING AMPLITUDE FUNCTIONS}

The starting point of the present method relies on the numerical integration of the independent solutions $u_{1}$ and $u_{2}$ of the Schrödinger equation (1). We choose $u_{1}$ and $u_{2}$ to be regular, respectively, at $s_{1}$ and $s_{2}$, so that $u_{1}\left(s_{1}\right)=0$, $u_{2}\left(s_{2}\right)=0$. Numerical integration proceeds through any standard method (e.g., by Numerov-Cooley integration or by the method of chasing) and we choose as the second boundary condition $u_{1}\left(t_{1}\right)>0$ and $u_{2}\left(t_{2}\right)>0$, where $t_{1}$ and $t_{2}$ are, respectively, the inner and outer turning points. $u_{2}(x)$ is then rescaled so that the Wronskian is set to $W=2 \sin \pi n(E) / \pi$ [indeed, if $u_{1}$ and $u_{2}$ are, respectively, regular at $s_{1}$ and $s_{2}$ then we must have $|W|=|2 I \sin \pi n(E)|[14]$; we then set $I$ $=\pi^{-1}$ and our sign convention accordingly]. At this point, we have potentially constructed not one but a family of amplitude functions given by Eq. (4): the reason is that any change $u_{1} \rightarrow u_{1} / b, u_{2} \rightarrow b u_{2}$, with $b$ real, gives the same Wronskian, but leads to different amplitude functions. Hence, since $I=\pi^{-1}$ and $c$ $=-\pi \cot \pi n(E) / 2$ the different amplitude functions depend on $b$ through

$$
\begin{aligned}
\alpha(x ; b)= & {\left[\frac{\pi}{2 \sin ^{2} \pi n(E)}\left(\frac{u_{1}^{2}(x)}{b^{2}}+b^{2} u_{2}^{2}(x)\right)\right.} \\
& \left.+\frac{\pi \cos \pi n(E)}{\sin ^{2} \pi n(E)} u_{1}(x) u_{2}(x)\right]^{1 / 2} .
\end{aligned}
$$

Of course, varying $b$ is tantamount to constructing different amplitude functions from solutions $u_{1}$ and $u_{2}$ having different boundary conditions at the turning points, but this does not concern us here: $u_{1}$ and $u_{2}$ are the numerically integrated (and to a certain extent arbitrary) functions.

The crucial observation is that there is one value of $b$, denoted $b_{o}$, for which $\alpha\left(x ; b_{o}\right)$ is the "optimal" amplitude function. $b_{o}$ is obtained by imposing

$$
\left.\frac{\partial \phi}{\partial b}\right|_{x=t_{2}}-\left.\frac{\partial \phi}{\partial b}\right|_{x=t_{1}}=0
$$

From Eq. (5), this condition-the extremalization of the quantum phase between the classical turning points-takes the form

$$
b_{o}^{2}=\left|\frac{u_{1}\left(t_{1}\right) u_{1}\left(t_{2}\right)}{u_{2}\left(t_{1}\right) u_{2}\left(t_{2}\right)}\right|^{1 / 2} .
$$

Hence $b_{o}$ is obtained by simply calculating the value of the numerical functions $u_{1}$ and $u_{2}$ at the classical turning points. $\alpha\left(x ; b_{o}\right)$ is the "optimal" nonoscillating amplitude function and its integral $\phi\left(x ; b_{o}\right)$ the corresponding "optimal" phase function. By construction the accumulated phase is $\beta(E)=\pi n(E)$, and the basis functions $f(x)$ and $g(x)$ of great use in scattering problems, given by $\{f, g\}$ $=\{\sqrt{2 I} \alpha(x) \sin \phi(x), \sqrt{2 I} \alpha(x) \cos \phi(x)\}$ are normalized to $I \pi \partial_{E} n$ (in both expressions, $I=\pi^{-1}$ ).

\section{Example 1: Harmonic oscillator}

We first illustrate our method on the harmonic oscillator. Here $k^{2}(x, E)=2\left(E-x^{2} / 2\right), s_{1}=-\infty, s_{2}=+\infty$. The relation $E_{0}=\nu+1 / 2$, where $\nu$ is the principal quantum number, is inverted to get $\nu(E)=E-1 / 2$, and the number of halfwavelengths is $n(E)=\nu(E)+1$. As a working example, for an energy corresponding to $\nu(E)=7.24$, we have integrated $u_{1}$ and $u_{2}$ with numerical values of $s_{1}$ and $s_{2}$ set as three times the turning points, and taking as second boundary conditions $u_{1}\left(t_{1}\right)=1$ and $u_{2}\left(t_{2}\right)=1$. We then divide $u_{2}(x)$ by $\approx-7.879$, so that the rescaled $u_{2}$ gives a Wronskian $W$ $=2 \sin 8.24 \pi / \pi$; the optimal amplitude-phase functions are then obtained for $b_{o}^{2} \simeq 7.88$. We have plotted in Fig. 1 the phase increment $\phi\left(t_{2} ; b\right)-\phi\left(t_{1} ; b\right)$ for different values of $b$ : the phase accumulation between the turning points is seen to have a minimum for $b=b_{o}$. 


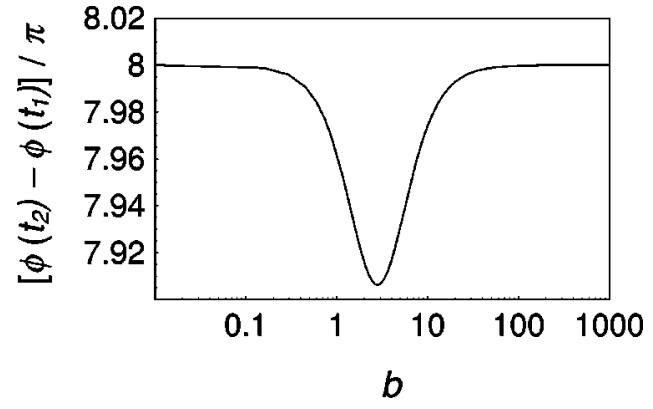

FIG. 1. The phase increment between the turning points $\phi\left(t_{2} ; b\right)-\phi\left(t_{1} ; b\right)$ for a harmonic oscillator with $\nu=7.24$ is plotted for different values of $b$ (note the logarithmic scale). The increment has a minimum for the optimal phase function, with $b=b_{o}$ (see text), and reaches the value of $8 \pi$ for $b \rightarrow 0$ or $\infty$ (whereas the total accumulated phase between $s_{1}$ and $s_{2}$ is $8.24 \pi$ ). The increment for the optimal phase function is not always a minimum, but can be a maximum as well, depending on the position of the zeros of $u_{2}$ relative to the turning points. However, the increment $\phi\left(t_{2}(E), E ; b_{o}(E)\right)-\phi\left(t_{1}(E), E ; b_{o}(E)\right)$ varies monotonically with the energy [and linearly with $n(E)]$.

\section{Example 2: Spherical harmonics}

Phase amplitude methods for spherical harmonics have seldom been developed (with the noteworthy exception of Ref. [4]). The usual equation for the associated Legendre function $\partial_{\theta}\left[\sin \theta \partial_{\theta} P_{l m}(\theta)\right] / \sin \theta+\left[l(l+1)-m^{2} \sin ^{-2} \theta\right] P_{l m}(\theta)$ $=0$ is put under the form

$$
\partial_{\theta}^{2} \chi_{l m}(\theta)+k_{l m}^{2}(\theta) \chi_{l m}(\theta)=0
$$

with $\chi_{l m}(\theta)=P_{l m}(\theta) \sin ^{1 / 2} \theta \quad$ and $\quad k_{l m}^{2}(\theta)=\left(l+\frac{1}{2}\right)^{2}-\left(m^{2}\right.$ $\left.-\frac{1}{4}\right) / \sin ^{2} \theta$. We have $s_{1}=0$ and $s_{2}=\pi$, and the relation $E_{0}(l) \equiv L^{2}=l(l+1)$ is inverted to give $l(E)=\frac{1}{2}(\sqrt{1+4 E}$ -1 ). Following our recipe (and keeping to the more standard notation $l$ for the total angular momentum effective quantum number, rather than $\nu$ ), the accumulated phase is now given by $\beta(E)=\pi n(E)$, with $n(E)=l-m+1$. Figure 2 displays rather than the amplitude the quantity $\alpha^{-2}\left(\theta ; b_{o}\right)$ for $l=13.7$ and $m=6$, which ascribes a total angular velocity (whereas for an arbitrary value of $b, \alpha(\theta)$ would display the nodal structure of the wave function). The pair of regular and

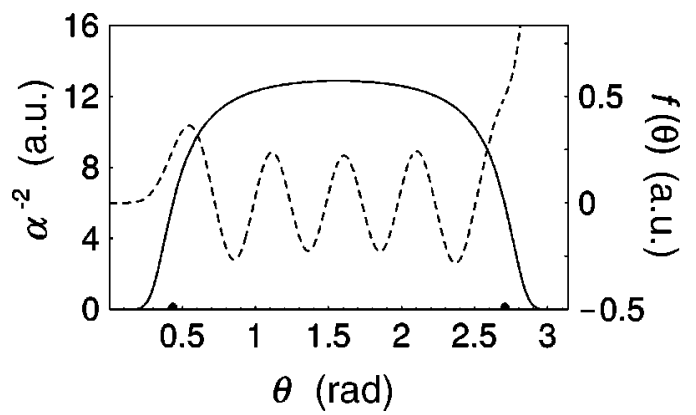

FIG. 2. The energy-normalized optimal function $\alpha^{-2}\left(\theta ; b_{o}\right)$ (smooth solid line, left scale) is plotted for $l=13.7, m=6$. The corresponding function regular at $s_{1}=0$ (see text) is represented by the dashed curve (right scale). The turning points are indicated on the $\theta$ axis. Atomic units (a.u.) are used.

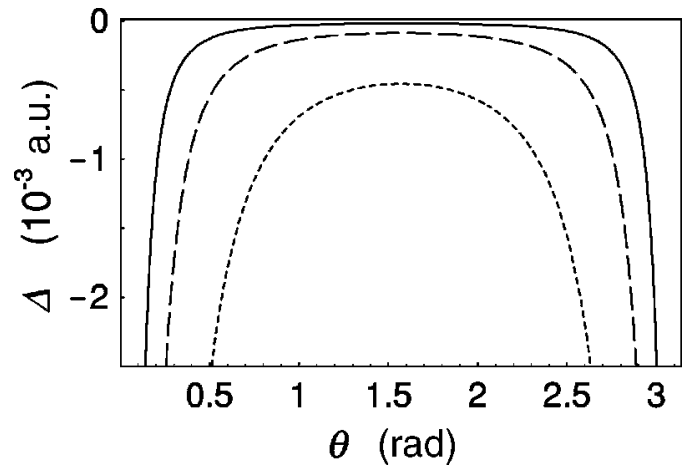

FIG. 3. $\Delta=(\pi \sin \theta / 2)^{-1 / 2}\left[\alpha_{l 0}\left(\theta ; b_{o}\right)-(l+1 / 2)^{-1 / 2}\right]$ is shown for three values of $l: l=6$ (dotted line), $l=12$ (dashed line), and $l$ $=22$ (solid line). The difference between the optimal amplitude function and the classical amplitude decreases as $l$ increases. The calculations were made by adding a small quantity $\varepsilon=10^{-6}$ to the integer value of $l$ for convergence purposes.

irregular functions, corresponding to $P_{l m}(\theta)$ when $l$ is an integer, is then retrieved by the standard formulas. The regular function, given by $f_{l m}(\theta)$ $=2^{1 / 2}(\pi \sin \theta)^{-1 / 2} \alpha_{l m}(\theta) \sin \phi_{l m}(\theta)$ is also shown on Fig. 2.

Note that when $m<2^{-1 / 2}$, which involves only a single but important quantized case, $m=0$, the effective potential does not have a minimum (but a metastable maximum) and our method cannot be applied since there are no turning points. However, we may take advantage of the symmetry of the potential and its derivative about $\theta=\pi / 2$; any $\theta<\pi / 2$ is mapped to $\pi-\theta>\pi / 2$, and, in particular, $s_{1}$ is mapped into $s_{2}$. Hence any couple of points $(\theta, \pi-\theta)$ can play the role of $t_{1}$ and $t_{2}$ in Eq. (12), and $b_{o}$ is accordingly obtained by solving

$$
b_{o}^{2}=\left|\frac{u_{1}(\theta) u_{1}(\pi-\theta)}{u_{2}(\theta) u_{2}(\pi-\theta)}\right|^{1 / 2},
$$

for any $\theta$. An example is shown in Fig. 3: we have plotted there the difference between the optimal amplitude $2^{1 / 2}(\pi \sin \theta)^{-1 / 2} \alpha_{l 0}\left(\theta ; b_{o}\right)$ and the semiclassical amplitude $2^{1 / 2}(\pi \sin \theta)^{-1 / 2}\left(l+\frac{1}{2}\right)^{-1 / 2}$. It can be seen that even for low values of $l$, the agreement is quite good.

\section{Example 3: Coulomb potential}

The centrifugal Coulomb potential problem has been arguably the main case study for amplitude-phase methods, in connection with short-range scattering in a long-range Coulomb field. Different methods [2-4,7-10] have been proposed to minimize the oscillations of the amplitude and phase functions, yielding satisfactory numerical results in practical computations. However, none of these methods led to a total suppression of the oscillations. Our recipe provides optimal amplitude and phase functions also in this case, provided Eq. (12) is implemented not with the momentum $k(x, E, l)=\sqrt{2}\left[E-l(l+1) /\left(2 x^{2}\right)+1 / x\right]^{1 / 2}$ appearing in the radial Schrödinger equation but with the modified wave number 


$$
k^{\delta}(x, E, l)=2^{1 / 2}\left[E-\frac{(l+\delta)^{2}}{2 x^{2}}+\frac{1}{x}\right]^{1 / 2},
$$

where $\delta$ is a transformation parameter arising from the singularity at $x=0$ which breaks the simple correspondence between the quantum wave number and the classical potential [15]. Employing the well-known Langer modification ( $\delta$ $=1 / 2$ ) leads to nonoscillating functions for sufficiently large quantum numbers but a correction must be implemented at low energies. The ansatz

$$
\delta(E, l)=\frac{1}{2}+\frac{\{(l+1) /[2(l+3)]\}^{\pi \ln (l+9 / 4)}}{\left[(-2 E)^{-1 / 2}-l-\frac{1}{2}+\sqrt{l(l+1)}\right]^{(l+1) / 2(l+3)}}
$$

was seen to efficiently correct $k(x, E, l)$ when using Eq. (11) even at low energies and $l$, and tends to the Langer modified momentum in the limit $E \rightarrow 0$ and/or $l \rightarrow \infty$.

To be precise, $u_{1}$ and $u_{2}$ are generated from the numerical integration of Eq. (1), i.e., with the momentum $k(x, E, l)$, and with numerical approximations to $s_{1}=0$ and $s_{2}=+\infty$. The eigenvalue relation $E_{0}=-1 /\left(2 \nu^{2}\right)$ is inverted to yield $n(E)=(-2 E)^{-1 / 2}-l$. The corrected momentum only enters in the calculation of the turning points, so that $t_{1}$ and $t_{2}$ in Eq. (12) correspond to the solutions of $k^{\delta}=0$, with $\delta$ given by Eq. (16). Note that the modified potential $k^{\delta}$ has a minimum for any positive value of $l$, so our method can also be applied to $l=0$. An illustration is given in Fig. 4 .

\section{DISCUSSION}

Relative to an arbitrary phase function, the optimal phase function $\phi_{o}(x, E)$ plays the role that the classical action $S(x, E)$ has vis-à-vis any other semiclassical phase function $\widetilde{\phi}_{o}(x, E): S$ is indeed the only nonoscillating semiclassical phase function [14]. Our method generates optimal functions by extremalizing $\phi_{o}(x, E)$ between two points related by a classical map; in this respect, we remark that quantum defect theory was recently reinterpreted as the realization of an exact quantum Poincaré map [16]. However, in most of the methods given in Refs. [1-4,6-8,10,11]—which were not able to totally eliminate the oscillations-classical mechanics intruded in the choice of the quantum amplitude and

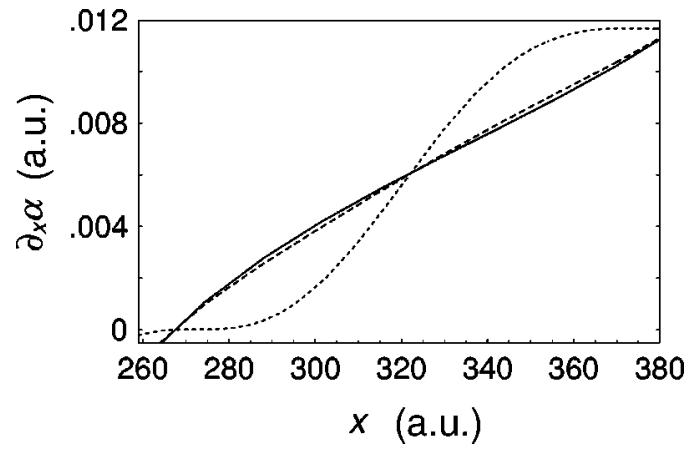

FIG. 4. The first derivative of the amplitude function for a centrifugal Coulomb potential ( $\nu=18.76, l=16)$ is plotted in a region around the bottom of the potential. The solid line results from following our prescription, whereas the dashed line almost superposed on the solid line has been calculated by employing the improved classical boundary conditions as given in Ref. [9]. For only slightly larger values of $n(E)=\nu-l$, both methods give the same nonoscillating amplitude function. On the other hand, plain (WKB) classical boundary conditions as used in [1,2] lead to oscillations, which are small relative to the oscillations displayed by an arbitrary amplitude function but are nevertheless clearly visible when plotting the first derivative (dotted line).

phase functions, usually by using a classical value as a boundary condition on Eq. (3). An improved and more efficient method due to Sidky [9] used a combination of classical boundary conditions so as to minimize $\partial_{x}^{2} k(x, E)^{-1 / 2}$, which appears as a driving force in a linearized equation for the difference between an "ideal" amplitude function and the classical amplitude $k(x, E)^{-1 / 2}$. For the harmonic oscillator and the Coulomb potential cases, the Sidky method and the present recipe yield quasi-identical nonoscillating amplitudephase functions; small differences were found only for small values of $n(E)$, as illustrated in Fig. 4. Another difference is that none of the other methods control the value of the accumulated phase $\beta(E)$ [which usually oscillates around the optimal value $\pi n(E)]$ whereas we impose through Eq. (8) $\beta(E)=\pi n(E)$ from the beginning. However, our method relies on integrating first the linear Eq. (1) rather than directly integrating the nonlinear Eq. (3), as in Ref. [9]; this may prove to be a drawback when working with potentials that extend to a very long range, as is the case for intramolecular potentials in cold atom collisions.
[1] H.J. Korsch and H. Laurent, J. Phys. B 14, 4213 (1981).

[2] C.H. Greene, A.R.P. Rau, and U. Fano, Phys. Rev. A 26, 2441 (1982).

[3] B. Yoo and C.H. Greene, Phys. Rev. A 34, 1635 (1986).

[4] F. Robicheaux, U. Fano, M. Cavagnero, and D.A. Harmin, Phys. Rev. A 35, 3619 (1987).

[5] W.E. Milne, Phys. Rev. 35, 863 (1930).

[6] E.Y. Sidky and I. Ben-Itzhak, Phys. Rev. A 60, 3586 (1999).

[7] F. Texier and C. Jungen, J. Phys. B 33, 2495 (2000).

[8] A. Matzkin, Phys. Rev. A 63, 012103 (2000).
[9] E.Y. Sidky, Phys. Essays 13, 489 (2000).

[10] M. Hiyama and M.S. Child, J. Phys. B 34, 3935 (2001).

[11] K.E. Thylwe and H.J. Korsch, J. Phys. A 34, 3497 (2001).

[12] H. Rosu and P. Espinoza, Phys. Rev. E 63, 037603 (2001).

[13] R.S. Kaushal, Class. Quantum Grav. 15, 197 (1998).

[14] A. Matzkin, J. Phys. A 34, 7833 (2001).

[15] M.V. Fedoriuk, Asymptotic Analysis (Springer, New York, 1993).

[16] F. Leyvraz, M. Lombardi, and T.H. Seligman, Phys. Lett. A 268, 309 (2000) 